\title{
Path Resummations and the Fermion Sign Problem
}

\author{
A. Alavi ${ }^{1}$ and A.J.W. Thom ${ }^{2}$
}

1 University of Cambridge, Chemistry Department, Lensfield Road, Cambridge CB2 1EW, U.K.

asa10@cam.ac.uk

2 University of Cambridge, Chemistry Department, Lensfield Road, Cambridge CB2 1EW, U.K.

ajwt3@cam.ac.uk

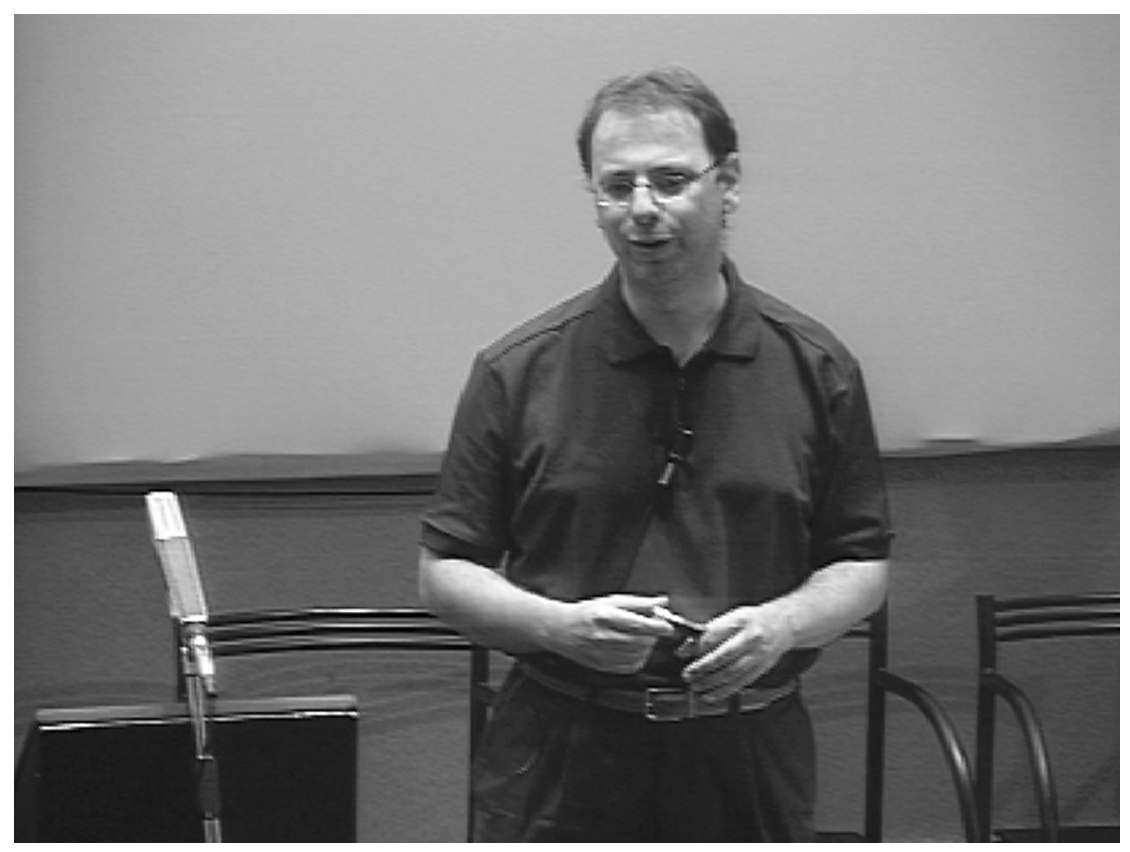

Ali Alavi 
686 A. Alavi and A.J.W. Thom

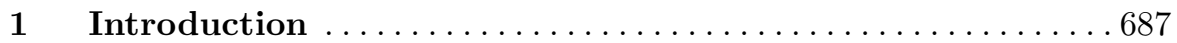

2 Graphs versus Paths . . . . . . . . . . . . . . . . . 689

3 A Useful Combinatorial Identity . . . . . . . . . . . . . 693

4 An Example: The Hubbard Model . . . . . . . . . . . . 696

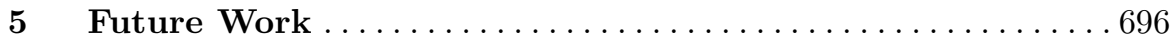

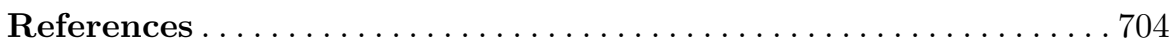


We review a recent method we have developed for Fermion quantum Monte Carlo. By using combinatorial arguments to perform resummations over paths, we reformulate the stochastic problem of sampling paths in terms of sampling "graphs", which are much better behaved with regards sign-cancellation problems encountered in path-integral simulations of Fermions. Detailed mathematical derivations of the new results are presented.

\section{Introduction}

In a recent paper [1], we have proposed a method to perform quantum Monte Carlo (QMC) simulations of Fermion systems based on the idea of sampling "graphs", rather than "paths". The latter, being rooted in Feynman's "realspace imaginary-time" path-integral (PI) theory [2-4], has been the paradigmatic approach to simulating quantum systems at finite temperature. For Fermion systems, however, there persists the infamous sign problem [5]. In the present contribution, we will review our new ideas, and in addition provide detailed mathematical derivations of theoretical results reported there, which we hope students may find useful. This paper is intended to be read in conjunction with [1].

Let us begin with a few words of motivation: why are we interested in developing QMC methods for treating electrons, when there exist highly accurate quantum chemical methods for computing electronic energies? The reason has to with the scaling of the algorithms with numbers of electrons. Wavefunction-based methods [6], such as Coupled-Cluster theory (CCSD $(T))$, which have emerged in the past few years as a "Rolls-Royce" method of obtaining accurate energies, scale as $\sim N^{6}-N^{7}$ ( $N$ is the number of electrons), which is prohibitively expensive. On the other hand, the ever-popular density functional methods, which have a highly favourable $N^{2}-N^{3}$ scaling, have important failings in treating strong-correlation systems, and non-local correlation phenomena such as van der Waals interactions. Quantum Monte Carlo methods, in principle, allow the way out, providing correlated energies with typically $\sim N^{3}$ scaling and, therefore, allow one to treat large systems whilst maintaining good accuracy. Currently the diffusion Monte Carlo method [7] within the fixed-node approximation is the most accurate of the QMC techniques. Attempts to go beyond the fixed-node approximation via the releasenode method [8] fail as the number of electrons increase.

In PI theory, the sign problem arises from the alternating sign of the weights of paths which exchange the positions of identical Fermions: odd and even permutations lead to negative and positive weights, respectively. More specifically, let us denote the spatial and spin coordinates of $N$ electrons with $\mathbf{X}=\left(\mathbf{x}_{1}, \ldots, \mathbf{x}_{N}\right)$. A given path can be described by a continuous function, $\mathbf{X}(\tau)$, with $\tau$ running from 0 to $\hbar \beta$. For equilibrium problems at finite temperature $k T=\beta^{-1}$, we are interested in paths which are closed, up to a permutation of the labels of the electrons: 


$$
\mathbf{X}(\hbar \beta)=\hat{P} \mathbf{X}(0)
$$

According to the principles of equilibrium quantum statistical mechanics [3], we associate with each such path a Boltzmann weight determined by the Hamiltonian of the system, multiplied by the sign of the permutation:

$$
\begin{aligned}
w[\mathbf{X}(\tau) ; \mathbf{X}, \hat{P} \mathbf{X}]= & \operatorname{sign}(\hat{P} \mathbf{X}) \times \\
& \left(\exp \left[-(1 / \hbar) \int_{0}^{\hbar \beta}\left(m|\dot{\mathbf{X}}|^{2} / 2+V[\mathbf{X}(\tau)]\right) d \tau\right]\right)_{\mathbf{X}(\hbar \beta)=\hat{P} \mathbf{X}(0)}
\end{aligned}
$$

The partition function is given by the path-integral of this weight over all such paths, for all permutations of end-points, and for all initial positions $\mathbf{X}$ :

$$
Q=\frac{1}{N !} \int d \mathbf{X} \sum_{\hat{P}} \int \mathcal{D} \mathbf{X}(\tau) w[\mathbf{X}(\tau) ; \mathbf{X}, \hat{P} \mathbf{X}]
$$

The expectation value of the energy can be found through the relation:

$$
E=-\frac{\partial \ln Q}{\partial \beta}
$$

and takes the form

$$
E=\frac{1}{Q} \frac{1}{N !} \int d \mathbf{X} \sum_{\hat{P}} \int \mathcal{D} \mathbf{X}(\tau) E[\mathbf{X}(\tau)] w[\mathbf{X}(\tau) ; \mathbf{X}, \hat{P} \mathbf{X}]
$$

where $E[\mathbf{X}(\tau)]$ is an energy estimator derived from the Hamiltonian of the system [9]. In numerical implementation, the continuous path is discretized into a number $P$ of time-slices:

$$
\mathbf{X}(\tau) \rightarrow \mathbf{X}_{1}, \mathbf{X}_{2} \ldots, \mathbf{X}_{P}, \hat{P} \mathbf{X}_{1} .
$$

Since in a Metropolis Monte Carlo simulation one can generate paths only with a positive probability proportional to $|w|$, in order to compute expectation values of the type (5) one has to resort to a potentially problematic expression:

$$
E=\frac{\langle\operatorname{sign}(w[\mathbf{X}]) E[\mathbf{X}]\rangle_{|w|}}{\langle\operatorname{sign}(w[\mathbf{X}])\rangle_{|w|}}
$$

Since $\operatorname{sign}(w)= \pm 1$, the denominator can be very poorly behaved. As the paths get longer (i.e. $P$ becomes large, which is necessary as $\beta$ becomes large), or as $N$ becomes large, one ends up sampling almost as many negative paths as positive ones, an exponential cancellation occurs in the sum over $\operatorname{sign}(w)$ of the generated paths, making this denominator all but impossible to estimate.

Our proposal as a way forward is to reformulate the problem so that the entities which we end up sampling are not individual paths, but rather integrated entities in which exponentially large numbers of paths have been 
summed over. It is reasonable to expect that these entities (which we call "graphs") will, as a result, be much better behaved with regards sign fluctuations, and therefore enable one to sample them stochastically. In our paper [1] we presented evidence for this assertion in the specific case of a dissociating $\mathrm{N}_{2}$ molecule. In this paper, we give another numerical example, a Hubbard model. In the next section, we first review the basic ideas of our approach.

\section{Graphs versus Paths}

In thinking about constructing paths in electron spaces, it is convenient from the outset to work in antisymmetrized spaces - conveniently done through the Slater Determinant formalism - and to construct paths in such spaces. Let us take a set of $2 M$ one-electron orthonormal spin-orbitals, $u_{1}(\mathbf{x}), u_{2}(\mathbf{x}) \ldots u_{2 M}(\mathbf{x})$, and denote a point in our $N$-electron space with $D_{\mathbf{i}}(\mathbf{X})$ (the label $\mathbf{i}$ being an ordered $N$-tuple of integers):

$$
D_{\mathbf{i}} \equiv D_{i_{1} i_{2} \ldots i_{N}}=\frac{1}{\sqrt{N !}} \operatorname{det}\left[u_{i_{1}} \ldots u_{i_{N}}\right],
$$

The orbitals can come from a variety of sources (e.g. they can be plane-waves, points on a regular 3D grid, or Hartree-Fock orbitals, Kohn-Sham orbitals etc). A given determinant describes an uncorrelated state of electrons, and in the limit of $M \rightarrow \infty$ this collection of $N_{\text {det }}=\left(\begin{array}{c}2 M \\ N\end{array}\right)$ Slater determinants could be used to expand any correlated wavefunction (satisfying the boundary conditions of the problem). Obviously one wants this basis to provide a good starting point to describe the physics of $N$ correlated electrons, and in common with wavefunction methods, the rate of convergence of our method will depend on the choice of this one-electron basis.

The advantage of using an antisymmetrized space is that one does not need to perform explicit averages over $N$ ! permuted paths - as implied by the sum $(1 / N !) \sum_{\hat{P}}$ in $(3)$. Instead, traces over this space are traces over purely antisymmetric parts, as is appropriate for a Fermion system:

$$
\begin{aligned}
Q & =\operatorname{Tr}\left[e^{-\beta \hat{H}}\right]=\sum_{\mathbf{i}}\left\langle D_{\mathbf{i}}\left|e^{-\beta \hat{H}}\right| D_{\mathbf{i}}\right\rangle \\
& =\sum_{\mathbf{i}_{1}} \sum_{\mathbf{i}_{2}} \ldots \sum_{\mathbf{i}_{P}}\left\langle D_{\mathbf{i}_{1}}\left|e^{-\beta \hat{H} / P}\right| D_{\mathbf{i}_{2}}\right\rangle\left\langle D_{\mathbf{i}_{2}}\left|e^{-\beta \hat{H} / P}\right| D_{\mathbf{i}_{3}}\right\rangle \ldots\left\langle D_{\mathbf{i}_{P}}\left|e^{-\beta \hat{H} / P}\right| D_{\mathbf{i}_{1}}\right\rangle
\end{aligned}
$$

A term in the above expansion corresponds to a closed path of $P$ steps in Slater determinant space:

$$
D_{\mathbf{i}_{1}} \rightarrow D_{\mathbf{i}_{2}} \rightarrow \ldots \rightarrow D_{\mathbf{i}_{P}} \rightarrow D_{\mathbf{i}_{1}}
$$

in a definite order; this is the analogue of a closed path as denoted in (6). The weight of such a path is: 


$$
\begin{aligned}
& w^{(P)}\left[\mathbf{i}_{1}, \mathbf{i}_{2}, \ldots, \mathbf{i}_{P}, \mathbf{i}_{1}\right]= \\
& \left\langle D_{\mathbf{i}_{1}}\left|e^{-\beta \hat{H} / P}\right| D_{\mathbf{i}_{2}}\right\rangle\left\langle D_{\mathbf{i}_{2}}\left|e^{-\beta \hat{H} / P}\right| D_{\mathbf{i}_{3}}\right\rangle \ldots\left\langle D_{\mathbf{i}_{P}}\left|e^{-\beta \hat{H} / P}\right| D_{\mathbf{i}_{1}}\right\rangle
\end{aligned}
$$

which is analogous to (2). As regards the sign-problem we have still not made much progress, $\operatorname{since} \operatorname{sign}\left(w^{(P)}\right)$ in $(10)$ is not positive definite, and indeed a very poorly behaved quantity since it depends on the product of the signs of $P$ factors, implying small variations in the path can produce wild fluctuations in the sign of the path.

Note there is no restriction that the elements along this path should be distinct. A given determinant can occur multiple times along a path. Suppose that this path consists of $n \leq P$ distinct determinants. This collection of determinants forms a $n$-vertex "graph" $G$ :

$$
G=\underbrace{\left\{D_{\mathbf{i}}, D_{\mathbf{j}}, D_{\mathbf{k}}, \ldots\right\}}_{n \text { determinants }}
$$

The order in which the determinants appear in the above is of no consequence. The connectivity of the graph is determined by $\rho_{\mathbf{i j}}=\left\langle D_{\mathbf{i}}\left|e^{-\beta \hat{H} / P}\right| D_{\mathbf{j}}\right\rangle . G$ is an object on which one can represent the paths which visit exclusively all vertices in $G$. The weight $w^{(n)}[G]$ of a given graph is obtained by summing over all such paths of length $P$ :

$$
w^{(n)}[G]=\sum_{\mathbf{i}_{1} \in G} \sum_{\mathbf{i}_{2} \in G} \sum_{\mathbf{i}_{3} \in G} \ldots \sum_{\mathbf{i}_{P} \in G}^{\prime} w^{(P)}\left[\mathbf{i}_{1}, \mathbf{i}_{2}, \ldots, \mathbf{i}_{P}, \mathbf{i}_{1}\right]
$$

The $/$ indicates that the summation indices $\mathbf{i}_{1}, \mathbf{i}_{2} \ldots \mathbf{i}_{P}$ are chosen from the set $G$ in such a way that each vertex in $G$ is visited at least once. This condition ensures that the weights of two different graphs $G_{a}$ and $G_{b}$ (i.e. two graphs which differ in at least one vertex) do not include the same paths which visit only the vertices of the set $G_{a} \cap G_{b}$ (See Fig. 1 in [1]). Therefore, the sum $w\left[G_{a}\right]+w\left[G_{b}\right]$ will not double-count the contribution of such paths. As a result, the partition function can be represented not as a "sum-over-paths" but rather a "sum-over-graphs":

$$
Q=\sum_{n} \sum_{G} w^{(n)}[G]
$$

which contains many fewer terms. It has been "contracted". In the above, the sum over $n$ represents a sum over 1-vertex, 2-vertex, 3-vertex etc., graphs, and for each $n$, the sum over $G$ represents the sum over all $n$-vertex graphs which can be constructed in the system. Since each graph represents a sum over exponentially large numbers of paths, it is reasonable to assume that the sign fluctuations of graphs will be better controlled than individual paths. There is, in addition, a more subtle reason why this contraction is beneficial, which has to do with the topology of graphs. Unlike paths, where there are equal number of odd and even permutations, certain graphs are positive definite 
and have no negative counterpart. Graphs which are trees (i.e. do not contain cycles) have positive weights. Any path on a tree which returns to its starting point must eventually retrace every outward step that it took, leading to a necessarily positive weight. Two-vertex graphs are the simplest and most important examples. If the terms with increasing $n$ have a reasonable convergence, so as to enable a truncation of the vertex series at some small order of $n$, the distribution of the significant graphs will be strongly biased towards the positive graphs, and enable a sampling of graphs without encountering an exponential cancellation.

Given the expression (13) for the partition function, one can express $E$ in a manner suitable for stochastic (Monte Carlo) method in which graphs are sampled with an appropriate probability and an energy estimator is averaged. Using (4), we find:

$$
E=\frac{1}{Q} \sum_{n} \sum_{G}-\frac{\partial \ln w^{(n)}[G]}{\partial \beta} \cdot w^{(n)}[G]
$$

Therefore, if graphs can be sampled with an unnormalised probability given by $w^{(n)}[G]$ (using a Metropolis Monte Carlo method), then the energy estimator becomes:

$$
\tilde{E}^{(n)}[G]=-\frac{\partial \ln w^{(n)}[G]}{\partial \beta}
$$

i.e.

$$
E=\left\langle\tilde{E}^{(n)}[G]\right\rangle_{w^{(n)}[G]}
$$

In other words, if on step $t$ of a Monte Carlo simulation consisting of $K$ steps one is at graph $G_{t}$, then the energy is given by the running average of $\tilde{E}^{(n)}[G]$ :

$$
E=\lim _{K \rightarrow \infty} \frac{1}{K} \sum_{t}^{K} \tilde{E}^{(n)}\left[G_{t}\right]
$$

In [1] we provide an algorithm to sample graphs generated according to a stochastic algorithm, and we will not elaborate further on this aspect.

Although (12)-(14) form the basis for a finite temperature method, it is possible (and indeed convenient) to modify the formalism to accelerate convergence towards the ground-state energy (the $\beta \rightarrow \infty$ limit), by ensuring that the graphs always contain a "good" determinant in them. For example, if we are using Hartree-Fock orbitals as the underlying one-particle basis, the Hartree-Fock determinant (composed out of the $N$ lowest energy orbitals), is usually a good starting point. In this case, the formalism is modified as follows. Let us denote the HF determinant as $D_{0}$ and consider:

$$
w_{0}=\left\langle D_{0}\left|e^{-\beta \hat{H}}\right| D_{0}\right\rangle
$$


and the function $\tilde{E}_{0}(\beta)$ :

$$
\tilde{E}_{0}(\beta)=-\frac{\partial \ln w_{0}}{\partial \beta}
$$

In [1], we show that $\tilde{E}_{0}$ is, as a function of $\beta$, bounded from above by the Hartree-Fock energy $E_{H F}$ and from below by the exact ground-state energy $E_{0}$ :

$$
\begin{gathered}
\lim _{\beta \rightarrow 0} \tilde{E}_{0}=E_{H F} \\
\lim _{\beta \rightarrow \infty} \tilde{E}_{0}=E_{0} \\
E_{0} \leq \tilde{E}_{0} \leq E_{H F}
\end{gathered}
$$

$w_{0}$ here plays the role of the partition function $Q$, since it can itself be expanded as a path-integral, with the proviso that, instead of performing a trace over all initial positions, one computes the sum over all paths of length $P$ which start and finish at $D_{0}$ :

$$
w_{0}=\sum_{\mathbf{i}_{1}} \sum_{\mathbf{i}_{2}} \ldots \sum_{\mathbf{i}_{P}}\left\langle D_{0}\left|e^{-\beta \hat{H} / P}\right| D_{\mathbf{i}_{1}}\right\rangle\left\langle D_{\mathbf{i}_{1}}\left|e^{-\beta \hat{H} / P}\right| D_{\mathbf{i}_{2}}\right\rangle \ldots\left\langle D_{\mathbf{i}_{P}}\left|e^{-\beta \hat{H} / P}\right| D_{0}\right\rangle
$$

Analogously to (13), we now seek to write:

$$
w_{0}=\sum_{n} \sum_{G} w_{0}^{(n)}[G]
$$

where $w_{0}^{(n)}[G]$ is defined analogously to (12), except for the fact that $D_{0}$ is considered the beginning and end point of paths:

$$
w_{0}^{(n)}[G]=\sum_{\mathbf{i}_{2} \in G} \sum_{\mathbf{i}_{3} \in G} \ldots \sum_{\mathbf{i}_{P} \in G}^{\prime} w^{(P)}\left[\mathbf{0}, \mathbf{i}_{2}, \ldots, \mathbf{i}_{P}, \mathbf{0}\right]
$$

In (25) it is required that $G$ contains $D_{0}$ among its members. The ' has the same meaning as before: all vertices in $G$ must be visited by the paths. Using (24) and (19) leads to an expression for $\tilde{E}_{0}$ suitable for a stochastic sampling of graphs, analogous to (15):

$$
\tilde{E}_{0}=\left\langle\tilde{E}_{0}^{(n)}[G]\right\rangle_{w_{0}^{(n)}[G]}
$$

or in the case where $w_{0}^{(n)}[G]$ is not positive-definite:

$$
\tilde{E}_{0}=\frac{\left\langle\operatorname{sign}\left(w_{0}^{(n)}[G]\right) \tilde{E}_{0}^{(n)}[G]\right\rangle_{|w|}}{\left\langle\operatorname{sign}\left(w_{0}^{(n)}[G]\right)\right\rangle_{|w|}}
$$


One might expect that the convergence of (24) with respect to the graph size $n$ to be reasonably rapid. Starting from the Hartree-Fock determinant, 2 -vertex graphs sum over paths which involve double-excitations, 3-vertex graphs include those with triple and quadruple excitations, and in general the $n$-vertex graph include paths with up to $2(n-1)$-fold excitations. From a physical view-point, it is reasonable to expect that a lot of correlation can be captured with fairly small graphs, in line with the experience from coupledcluster and perturbation theories. Here the inverse-temperature-like parameter $\beta$ plays a useful role. As we show for the $\mathrm{N}_{2}$ molecule in [1], for small $\beta$, the convergence with respect to $n$ is rapid, since at small $\beta$, the paths cannot move far from $D_{0}$. At large $\beta$ the convergence at a given graph-size will be poor, and unphysical results will ensue. However, one get meaningful results at an intermediate $\beta$ before convergence breakdown.

\section{A Useful Combinatorial Identity}

How can one compute terms in $w^{(n)}[G]$ in (13) (and $w_{0}^{(n)}[G]$ in $(24)$ ), which are necessary to do the Monte Carlo sampling? One can get an intuitive sense of this by rearranging the terms in the partition function to correspond with paths with increasing numbers of "hops":

$$
\begin{aligned}
& Q=\sum_{\mathbf{i}}\left[\rho_{\mathbf{i i}}{ }^{P}+\sum_{\mathbf{j}}^{\mathbf{i}} \sum_{n=0}^{P-2} \sum_{m=0}^{P-2-n} \rho_{\mathbf{i i}}{ }^{n} \rho_{\mathbf{i j}} \rho_{\mathbf{j} \mathbf{j}}{ }^{m} \rho_{\mathbf{j i}} \rho_{\mathbf{i i}}{ }^{P-2-m-n}\right. \\
& \left.+\sum_{\mathbf{j}}^{\prime \mathbf{i}} \sum_{\mathbf{k}}^{\mathbf{i} \mathbf{j} \mathbf{j}} \sum_{n=0}^{P-3} \sum_{m=0}^{P-3-n} \sum_{l=0}^{P-3-n-m} \rho_{\mathbf{i i}}{ }^{n} \rho_{\mathbf{i j}} \rho_{\mathbf{j} \mathbf{j}}{ }^{m} \rho_{\mathbf{j} \mathbf{k}} \rho_{\mathbf{k k}}{ }^{l} \rho_{\mathbf{k i}} \rho_{\mathbf{i i}}{ }^{P-3-n-m-l}+\ldots\right]
\end{aligned}
$$

where we have set $\rho_{\mathbf{i j}} \equiv\left\langle D_{\mathbf{i}}\left|e^{-\beta \hat{H} / P}\right| D_{\mathbf{j}}\right\rangle$. Noting that we are getting nested sums, it proves useful to define a function $Z_{h}^{(P)}\left(x_{1}, x_{2}, \ldots, x_{h}\right)$ as follows:

$$
Z_{h}^{(P)}\left(x_{1}, x_{2}, \ldots, x_{h}\right)=\sum_{n_{1}=0}^{P-h} \sum_{n_{2}=0}^{P-h-n_{1}} \ldots \sum_{n_{h}=0}^{P-h-\sum_{i=1}^{h-1} n_{i}} x_{1}^{n_{1}} x_{2}^{n_{2}} \ldots x_{h}^{n_{h}}
$$

We will shortly discuss the properties of such nested sums. Let us for the moment note that we can cast each term in the square-brackets of (28) (which we define as $w_{\mathbf{i}}$ ) as follows: 


$$
\begin{aligned}
w_{\mathbf{i}}= & \rho_{\mathbf{i i}}{ }^{P}\left(1+\sum_{\mathbf{j}}^{\prime \mathbf{i}} \frac{\rho_{\mathbf{i j}} \rho_{\mathbf{j i}}}{\rho_{\mathbf{i i}}{ }^{2}} Z_{2}^{(P)}\left(1, \frac{\rho_{\mathbf{j} \mathbf{j}}}{\rho_{\mathbf{i i}}}\right)\right. \\
& +\sum_{\mathbf{j}}^{\prime \mathbf{i}} \sum_{\mathbf{k}}^{\prime \mathbf{j}, \mathbf{i}} \frac{\rho_{\mathbf{i j}} \rho_{\mathbf{j k}} \rho_{\mathbf{k i}}}{\rho_{\mathbf{i} \mathbf{i}}{ }^{3}} Z_{3}^{(P)}\left(1, \frac{\rho_{\mathbf{j} \mathbf{j}}}{\rho_{\mathbf{i i}}}, \frac{\rho_{\mathbf{k k}}}{\rho_{\mathbf{i i}}}\right) \\
& \left.+\sum_{\mathbf{j}}^{\prime \mathbf{i}} \sum_{\mathbf{k}}^{\prime \mathbf{j}} \sum_{\mathbf{l}}^{\prime \mathbf{k}, \mathbf{i}} \frac{\rho_{\mathbf{i j}} \rho_{\mathbf{j k}} \rho_{\mathbf{k l}} \rho_{\mathbf{l i}}}{\rho_{\mathbf{i} \mathbf{i}}{ }^{4}} Z_{4}^{(P)}\left(1, \frac{\rho_{\mathbf{j} \mathbf{j}}}{\rho_{\mathbf{i i}}}, \frac{\rho_{\mathbf{k} \mathbf{k}}}{\rho_{\mathbf{i} \mathbf{i}}}, \frac{\rho_{\mathbf{l l}}}{\rho_{\mathbf{i i}}}\right)+\ldots\right)
\end{aligned}
$$

In the sums above, each sum is prime with respect to (i.e. excludes) the index of the previous sum, except for the inner-most sum, which is also prime with respect to i. For example, in the 4 -hop term, $\mathbf{k}$ can equal $\mathbf{i}$, but not $\mathbf{j}$. Similarly, $\mathbf{l}$ can equal $\mathbf{j}$, but not $\mathbf{k}$ or $\mathbf{i}$.

Physically, $Z_{h}^{(P)}$ is a combinatorial factor which accounts for the number of ways the $P-h$ "stay-put" terms can be arranged for a given number of hops $h$. The rationale for this type of expansion is due to the diagonal dominance of the $\rho_{\mathbf{i j}}$ matrix. Every time we hop, we incur a penalty; therefore paths which involve many hops each carry an exponentially decreasing weight. On the other hand, the number of such paths increases exponentially. Therefore, the rate of convergence of the expansion above is difficult to predict. At high temperatures (or weak coupling) it will be fast (the off-diagonal $\rho_{\mathbf{i j}}$ are small), and otherwise slow. Our aim will be further summations in (30) to produce a rapidly convergent series.

In Appendix A, we prove the following identity:

$$
Z_{h}^{(P)}\left(x_{1}, \ldots, x_{h}\right)=\frac{1}{2 \pi i} \oint_{C} \frac{z^{P}-1}{(z-1) \prod_{\alpha}\left(z-x_{\alpha}\right)} d z
$$

where $C$ is a contour which encloses the poles $\left(x_{1}, \ldots, x_{h}\right)$. This integral has some remarkable properties discussed in the Appendix. Crucially, it enables us to sum over paths with different numbers of hops. For example, consider a 3-cycle $\mathbf{i} \rightarrow \mathbf{j} \rightarrow \mathbf{k} \rightarrow \mathbf{i}$ involving the transition matrix elements $\rho_{\mathbf{i j}} \rho_{\mathbf{j k}} \rho_{\mathbf{k i}}$. This 3-cycle (whose weight can be negative), will be involved in the following 3-hop, 6-hop,..., paths which form a typical alternating series:

$$
S=\frac{\rho_{\mathbf{i j}} \rho_{\mathbf{j k}} \rho_{\mathbf{k i}}}{\rho_{\mathbf{i i}}{ }^{3}} Z_{3}+\left(\frac{\rho_{\mathbf{i j}} \rho_{\mathbf{j k}} \rho_{\mathbf{k i}}}{\rho_{\mathbf{i i}}{ }^{3}}\right)^{2} Z_{6}+\cdots
$$

Defining the primitive cycle function:

$$
A_{\mathbf{i j k}}(z)=\frac{\rho_{\mathbf{i j}} \rho_{\mathbf{j k}} \rho_{\mathbf{k i}}}{\left(z \rho_{\mathbf{i i}}-\rho_{\mathbf{i i}}\right)\left(z \rho_{\mathbf{i i}}-\rho_{\mathbf{j} \mathbf{j}}\right)\left(z \rho_{\mathbf{i i}}-\rho_{\mathbf{k k}}\right)}
$$

we obtain:

$$
S=\sum_{n} \frac{1}{2 \pi i} \oint_{C} \frac{z^{P}-1}{z-1}\left[A_{\mathbf{i j k}}(z)\right]^{n} d z
$$


Switching the order of summation and integration, we obtain:

$$
S=\frac{1}{2 \pi i} \oint_{C} \frac{z^{P}-1}{z-1} \frac{1}{1-A_{\mathrm{ijk}}(z)} d z
$$

Since $1-A_{\mathbf{i j k}}(z)=0$ is a cubic equation in $z$, it has three roots, which can be used to evaluate (34) in terms of three residues - see Appendix A.

The above is a simple example of the analytic summation over an alternating series. One can similarly treat more complex paths, which consist of sums of combinations of cyclic paths. In [1], we derive in detail sums over paths (which start and finish at $\mathbf{i}$ ) constructed on two elemental types of graphs star-graphs and chain-graphs. We quote below the results:

$$
\begin{aligned}
S_{\mathbf{i}}^{\text {star }} & =\frac{1}{2 \pi i} \oint_{C} \frac{z^{P}-1}{z-1} \frac{1}{\left(1-A_{G_{1}}-A_{G_{2}}-\cdots-A_{G_{g}}\right)} d z \\
S_{\mathbf{i}}^{\text {chain }} & =\frac{1}{2 \pi i} \oint_{C} \frac{z^{P}-1}{z-1} \frac{1}{1-\frac{A_{G_{1}}}{1-\frac{A_{G_{2}}}{1-\cdots}}} d z
\end{aligned}
$$

In the above, $A_{G_{1}}, A_{G_{2}}, \ldots$ are primitive cycle functions from which the stargraphs and chain-graphs are constructed.

Using these, we show that paths constructed on the general 3-vertex graph (which start and finish at i) contribute:

$$
\begin{aligned}
S_{\mathbf{i}}^{(3)}[\{\mathbf{i}, \mathbf{j}, \mathbf{k}\}] & =\frac{1}{2 \pi i} \oint_{C} \frac{z^{P}-1}{z-1} \frac{1}{1-\frac{A_{\mathbf{i j}}}{1-A_{\mathbf{j k}}}-\frac{A_{\mathbf{i k}}}{1-A_{\mathbf{j k}}}-2 \frac{A_{\mathbf{i j k}}}{1-A_{\mathbf{j k}}}} d z \\
& =\frac{1}{2 \pi i} \oint_{C} \frac{z^{P}-1}{z-1} \frac{1-A_{\mathbf{j k}}}{1-A_{\mathbf{i j}}-A_{\mathbf{i k}}-A_{\mathbf{j k}}-2 A_{\mathbf{i j k}}} d z
\end{aligned}
$$

The denominator of the three-vertex term, $1-A_{\mathbf{i j}}-A_{\mathbf{i k}}-A_{\mathbf{j k}}-2 A_{\mathbf{i j k}}=0$, reduces to a cubic polynomial in $z$, which can be solved to yield three roots, which are in turn used to evaluated the contour integral using the residue theorem.

By a similar technique of enumerating the distinct circuits on a complete 4-vertex graph, one can construct the unfolded representation and hence evaluate $S_{\mathbf{i}}^{(4)}[\{\mathbf{i}, \mathbf{j}, \mathbf{k}, \mathbf{l}\}]$. This procedure is carried out in the Appendix B. Although the resulting expression is quite unwieldy, the final form simplifies remarkably to a quartic polynomial on the denominator of the contour-integral.

The final expression for the weights for graphs with 2, 3 and 4 vertices are given by (46-48) of [1], and they are not repeated here. These take into account a double-counting corrections as explained there. We are optimistic to be able to generalise our method to larger graphs. 


\section{An Example: The Hubbard Model}

We demonstrate some of the ideas for a $\sqrt{18} \times \sqrt{18}$ Hubbard lattice containing 18 electrons, with periodic boundary conditions. This model has been extensively studied [11]. The Hubbard parameter was set to $U / t=4$. The groundstate of the non-interacting system, being diagonalised by plane-waves, is a closed shell consisting of $1+4+4$ doubly occupied levels. There are $2 M=36$ one particle orbtials, leading to $N_{\text {det }}=9075135300$ determinants for an $N=18$ system. There is a unique Fermi determinant. The matrix elements of the high-temperature density matrix $\rho_{\mathbf{i j}}$ were computed according to a Trotter decompostion formula ((30) of [1]). Corrections to this formula go as $(\beta / P)^{2}$, and are negligible for sufficiently small $\beta / P$ (we used $\beta / P=10^{-4}$ ). In addition, matrix elements with magnitude smaller $10^{-6}$ were set to zero, which represented a useful (factor of 2) but not essential saving in computer time. It is important to note that matrix elements between determinants which are more than double excitations away from each other, are zero. In addition, single excitations do not couple in this system due to translational invariance (momentum conservation).

We computed the weights of all graphs which include the Fermi determinant, up to 4-vertices. Graphs are classified according to whether they were trees or cyclic graphs. In Table 1, we give the total number of graphs which arise in these two catagories, and their integrated weight. In addition, the energies as measured by $\tilde{E}_{0}(19)$ are given for each vertex size truncation. The first observation is that there is an (expected) explosive growth in the number of graphs as the graph size $n$ increases - by roughly 3 orders of magnitude from each increment in $n$. It is significant that the number of tree graphs remain overwhelmingly dominant even at the 4 -vertex level, as do the relative weights of the tree graphs compared to cyclic graphs. Since the former are positive definite, this implies that a stochastic sampling of graphs would not encounter a serious sign-problem. The relative magnitude of the contributions of the 3 different graph sizes is $\beta$ dependent. At $\beta=1$, the 3 -vertex graphs contribute the most to the overall weight, whereas the $\beta=5$ the 4 -vertex graphs are significant. This indicates that the convergence of the vertex series is strongly $\beta$ dependent. This is also shown in the energies. Although at both $\beta$ the energies decrease with increasing graph-size, the convergence is much faster at $\beta=1$. The exact ground-state energy is known to be -17.25239 , whilst the Hartree-Fock energy is -14 . Therefore, at $\beta=1$, the $\tilde{E}_{0}$ at the 4 vertex level captures some $65 \%$ of the ground-state correlation energy, whilst at $\beta=5$ the result is somewhat worse (only 18\%). This indicates that, for highly accurate calculations, we will need to go beyond the 4-vertex graphs.

\section{Future Work}

Although our method is very much in its infancy, we believe our ideas can be developed and usefully applied to many types of electronic problems. Current 
Table 1. Number and the natural logarithm of the weights of graphs for a Hubbard model. The energies (in units of the Hubbard $t$ ) are also reported.

\begin{tabular}{|c|c|c|c|c|c|}
\hline \multirow{3}{*}{ vertex } & \multicolumn{4}{|c|}{$\beta=1$} & \multirow{3}{*}{$\tilde{E}_{0}$} \\
\hline & \multicolumn{2}{|c|}{ trees } & \multicolumn{2}{|c|}{ cyclic } & \\
\hline & number & $\ln w_{0}^{(n)}[G]$ & number & $\mid \ln w_{0}^{(n)}[G$ & \\
\hline 2 & 425 & 2.6174 & 0 & - & -14.8593 \\
\hline 3 & 248524 & 2.8557 & 4064 & 0.1497 & -15.5963 \\
\hline 4 & 166591656 & 1.7845 & 6222444 & 0.7217 & -16.1097 \\
\hline \multirow{3}{*}{ vertex } & \multicolumn{4}{|c|}{$\beta=5$} & \multirow{3}{*}{$\widetilde{E}_{0}$} \\
\hline & & ees & & clic & \\
\hline & number & $\ln w_{0}^{(n)}[G]$ & number & $\mid \ln w_{0}^{(n)}[G]$ & \\
\hline 2 & 425 & 15.3019 & $\overline{0}$ & - & -14.1977 \\
\hline 3 & 248484 & 108.0713 & 4064 & 5.3550 & -14.3978 \\
\hline 4 & 166591656 & 472.057 & 6222444 & 75.3802 & -14.5974 \\
\hline
\end{tabular}

work is to calibrate the method against the benchmark (Pople) set of molecules, which we hope to report on in the near future. It is an extremely exciting prospect to perform for Fermion systems a simulation which is stable, albeit approximate, through the truncation of of the graph-expansion of the partition function. We are currently working on the extension of the method to larger graphs. There are equally important issues of a more technical nature which will also need attention. A key property is the favourable scaling of the method: apart from an initialisation step of computing the 4-index integrals (itself an $N^{4} M$ step), the calculation of the weights is an $N^{2}$ process. The main bottleneck very rapidly becomes the initialisation step if all 4-index integrals are calculated. A work-around this problem will become necessary if we are to tackle large systems consisting of hundreds of electrons, but we believe this issue will be resolvable through on-the-fly calculation and storage/retrieval of the integrals.

We are grateful for the support of the EPSRC through a Portfolio Award.

\section{Appendix A: Proof of (31)}

Consider the multiple nested-sum:

$$
Z_{h}^{(P)}\left(x_{1}, \ldots, x_{h}\right)=\sum_{n_{1}=0}^{P-h} \sum_{n_{2}=0}^{P-h-n_{1}} \ldots \sum_{n_{h}=0}^{P-h-\sum_{i}^{h-1} n_{i}} x_{1}^{n_{1}} x_{2}^{n_{2}} \ldots x_{h}^{n_{h}}
$$

This sum is non-zero only for $P \geq h$. For $P<h, Z_{h}$ is identically zero for all $\left\{x_{i}\right\}$. We shall also assume that the $\left\{x_{i}\right\}$ can take arbitrary values, and in particular, that they can be "degenerate", i.e. some or all of them can be equal. 
By induction, we would like to prove:

$$
Z_{h}^{(P)}\left(x_{1}, \ldots, x_{h}\right)=\frac{1}{2 \pi i} \oint_{C} \frac{z^{P}-1}{(z-1) \prod_{j=1}^{h}\left(z-x_{j}\right)} d z
$$

where the contour $C$ encloses $x_{1} \ldots, x_{h}$. The case $h=1$ is immediately verified:

$$
\begin{aligned}
Z_{1}^{(P)}(x) & =\frac{1}{2 \pi i} \oint_{C} \frac{z^{P}-1}{(z-1)(z-x)} d z \\
& =\frac{x^{P}-1}{x-1}=\sum_{n=0}^{P-1} x^{n}
\end{aligned}
$$

Now assume (39) holds for $h$, and consider $h+1$.

$$
\begin{aligned}
& Z_{h+1}^{(P)}\left(x_{1}, \ldots, x_{h}, x_{h+1}\right)=\sum_{n=0}^{P-h-1} x_{h+1}^{n} \sum_{n_{1}=0}^{P-h-1-n} \ldots \sum_{n_{h}=0}^{P-h-1-n-\sum_{i} n_{i}} x_{1}^{n_{1}} \ldots x_{h}^{n_{h}} \\
&=\sum_{n=0}^{P-h-1} x_{h+1}^{n} Z_{h}^{(P-n-1)}\left(x_{1}, \ldots, x_{h}\right) \\
&=\sum_{n=0}^{P-1} x_{h+1}^{n} Z_{h}^{(P-n-1)}\left(x_{1}, \ldots, x_{h}\right) \\
&=\sum_{n=0}^{P-1} x_{h+1}^{n} \frac{1}{2 \pi i} \oint_{C} \frac{z^{P-n-1}-1}{(z-1) \prod_{i}^{h}\left(z-x_{i}\right)} d z \\
&= \frac{1}{2 \pi i} \oint_{C} \frac{\sum_{n=0}^{P-1}\left(x_{h+1} / z\right)^{n} z^{P-1}-x_{h+1}^{n} d z}{(z-1) \prod_{i}^{h}\left(z-x_{i}\right)} \\
&=\frac{1}{2 \pi i} \oint_{C} \frac{\left(z^{P}-x_{h+1}^{P}\right) /\left(z-x_{h+1}\right)-\left(1-x_{h+1}^{P}\right) /\left(1-x_{h+1}\right)}{(z-1) \prod_{i}^{h}\left(z-x_{i}\right)} d z \\
&=\frac{1}{2 \pi i} \oint_{C} \frac{1}{(z-1) \prod_{i}^{P+1}\left(z-x_{i}\right)} d z-\frac{1}{2 \pi i} \oint_{C} \frac{1-x_{h+1}^{P}}{(z-1)\left(1-x_{h+1}\right) \prod_{i}^{h}\left(z-x_{i}\right)} d z \\
&=\frac{1}{2 \pi i} \oint_{C} \frac{\left(z^{P}-x_{h+1}^{P}-x_{h+1} z^{P}+x_{h+1}^{P+1}-z+x_{h+1}+x_{h+1}^{P} z-x_{h+1}^{P+1}-1+1\right.}{(z-1)\left(1-x_{h+1}\right) \prod_{i}^{h+1}\left(z-x_{i}\right)} d z
\end{aligned}
$$

where in the last line we have added \pm 1 to the numerator. Continuing:

$$
\begin{aligned}
& =\frac{1}{2 \pi i} \oint_{C} \frac{(z-1)\left(x_{h+1}^{P}-1\right)-\left(z^{P}-1\right)\left(x_{h+1}-1\right)}{(z-1)\left(1-x_{h+1}\right) \prod_{i}^{h+1}\left(z-x_{i}\right)} d z \\
& =\frac{1}{2 \pi i} \oint_{C} \frac{\left(x_{h+1}^{P}-1\right)}{\left(1-x_{h+1}\right) \prod_{i}^{h+1}\left(z-x_{i}\right)} d z+\frac{1}{2 \pi i} \oint_{C} \frac{\left(z^{P}-1\right)}{(z-1) \prod_{i}^{h+1}\left(z-x_{i}\right)} d z
\end{aligned}
$$


Next, we use the identity [10]:

$$
\frac{1}{2 \pi i} \oint_{C} \frac{1}{\prod_{i}^{h+1}\left(z-x_{i}\right)} d z=0 \text { for } h \geq 1
$$

which can be verified by considering the Laurent expansion of the integrand:

$$
\begin{aligned}
\frac{1}{z^{h+1} \prod_{i}^{h+1}\left(1-x_{i} / z\right)}= & z^{-h-1}+\left(x_{1}+\ldots+x_{h+1}\right) z^{-h-2} \\
& +\left(x_{1}^{2}+x_{1} x_{2}+\ldots\right) z^{-h-3}+\ldots
\end{aligned}
$$

Integrating term by term, everything vanishes if $h \geq 1$, which by hypothesis is the case. The first term in (41) is therefore zero, leaving:

$$
Z_{h+1}^{(P)}\left(x_{1}, x_{2}, \ldots, x_{h+1}\right)=\frac{1}{2 \pi i} \oint_{C} \frac{\left(z^{P}-1\right)}{(z-1) \prod_{i}^{h+1}\left(z-x_{i}\right)} d z
$$

which is precisely the required result.

Incidently, the property that the contour-intergal formula (39) respects the identity $Z_{h}^{(P)}=0$ for $h>P$, for any set of arguments $\left\{x_{i}\right\}$, follows from a generalisation of (42), namely:

$$
\frac{1}{2 \pi i} \oint_{C} \frac{z^{P}}{\prod_{i}^{h+1}\left(z-x_{i}\right)} d z=0 \text { for } P<h
$$

which can be proven using the Laurent expansion of the integrand and integrating term-by-term, as above. This allows one to replace all summations over $h$ to infinity, thereby considerably simplifying the manipulation of sums.

If the $x_{1} \ldots, x_{h}$ are all distinct, the residue theorem gives:

$$
Z_{h}^{(P)}\left(x_{1}, \ldots, x_{h}\right)=\sum_{i=1}^{h} \frac{x_{i}^{P}-1}{\left(x_{i}-1\right) \prod_{j \neq i}\left(x_{i}-x_{j}\right)} .
$$

If there are multiple poles, then the appropriate version of the residue theorem needs to be applied, viz:

$$
\frac{1}{2 \pi i} \oint_{C} f(z) d z=(\text { sum of enclosed residues })
$$

where a residue for a pole of order $m$ at $z_{0}$ is given by:

$$
\frac{1}{(m-1) !} \frac{d^{m-1}}{d z^{m-1}}\left[\left(z-z_{0}\right)^{m} f(z)\right]
$$

(46) has been derived by Hall [12] by an independent method. 


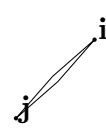

. $\mathrm{k}$
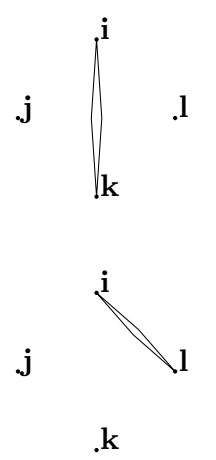

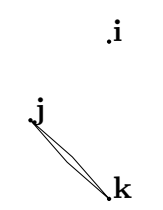

.1
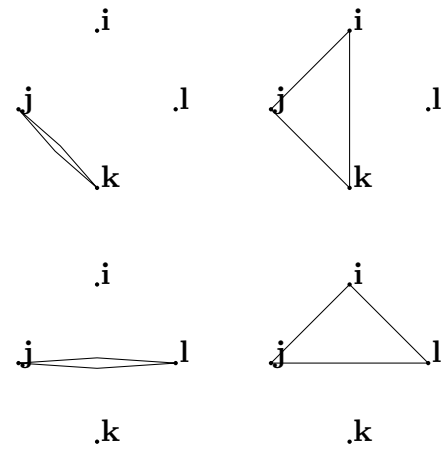

.i

j
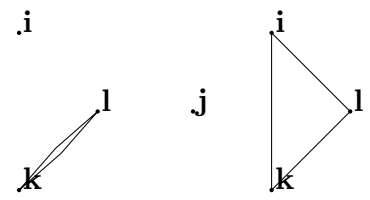
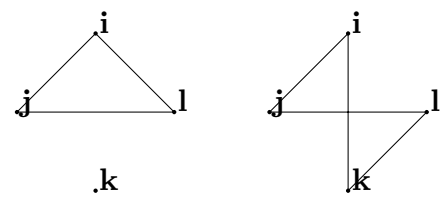

j

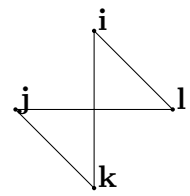

i

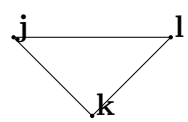

Fig. 1. All 13 distinct four-vertex primitive cycles

\section{Appendix B: The Complete 4-vertex Graph}

With the insight gained from constructing the complete three-vertex graph, we now develop an algorithm to create a graph constructed of primitive circuits linked in the form of chain and star graphs. Once such a graph has been constructed, it is a simple matter to write down the expression about which the contour must be taken to sum all possible graphs.

For a $n$-vertex graph, we must first list all possible primitive circuits. Here we give as an example the four-vertex graph, which has 20 primitive circuits (Fig. 1):

$$
\begin{array}{r}
(\mathbf{i j}),(\mathbf{i k}),(\mathbf{i l}),(\mathbf{j k}),(\mathbf{j l}),(\mathbf{k l}),(\mathbf{i j k}),(\mathbf{i k j}),(\mathbf{i j l}),(\mathbf{i l j}),(\mathbf{i k l}),(\mathbf{i l k}),(\mathbf{j k l}),(\mathbf{j l k}) \\
(\mathbf{i j k l}),(\mathbf{i l k j}),(\mathbf{i j l k}),(\mathbf{i k l j}),(\mathbf{i k j l}),(\mathbf{i l j k})
\end{array}
$$

Of these, thee are four which do not contain the pivot $\mathbf{i}$, and the remaining 16 do.

The algorithm to construct the complete $n$-vertex graph is as follows:

1. Set the current pivot to $\mathbf{i}$, the global pivot. 
2. Take the current list of primitive graphs, and create a star graph consisting of all of the primitive graphs which contain the current pivot.

3. Recurse through each of the primitive graphs we have just added. For each vertex of each graph, repeat from step 2, having eliminated all graphs containing vertices between the current pivot and the vertex we are considering.

We give as an example the $n$-vertex algorithm applied to the four-vertex graph.

Setting $\mathbf{i}$ as our current pivot, the list of all graphs containing $\mathbf{i}$ is

$$
\begin{array}{r}
(\mathbf{i j}),(\mathbf{i k}),(\mathbf{i l}),(\mathbf{i j k}),(\mathbf{i k j}),(\mathbf{i j l} \mathbf{l}),(\mathbf{i l j}),(\mathbf{i k l}),(\mathbf{i l k}) \\
(\mathbf{i j k} \mathbf{k}),(\mathbf{i l k j}),(\mathbf{i j l k}),(\mathbf{i k l j}),(\mathbf{i k j l}),(\mathbf{i l j k})
\end{array}
$$

Forming a star graph with all of these graphs pivoting and joining at $\mathbf{i}$ we result in Fig. 3. We now consider the two-, three- and four-vertex parts of this star graph.

Starting with (ij), we take $\mathbf{j}$ as the new pivot, we must now exclude all primitive graphs including $\mathbf{i}$. From this, the list of graphs containing the new pivot $\mathbf{j}$ is,

$$
(\mathbf{j k}),(\mathbf{j} \mathbf{l}),(\mathbf{j k} \mathbf{l}),(\mathbf{j} \mathbf{l k})
$$

A star graph is fomed by joining all these graphs at $\mathbf{j}$. Now for each of these subgraphs, vertices $\mathbf{i}$ and $\mathbf{j}$ have been traversed, so the only remaining graph without these is $(\mathbf{k l})$. Taking each of the graphs, we follow the same procedure, attaching a $(\mathbf{k l})$ to the $(\mathbf{j k})$ and an $(\mathbf{l k})$ to the $(\mathbf{j} \mathbf{l})$. Looking at the $(\mathbf{j k} \mathbf{k})$ graph, we choose $\mathbf{k}$ as the next pivot. The only graph available to us is a $(\mathbf{k l})$, which we attach. Similarly, we attach a (lk) to the $\mathbf{l}$ of $(\mathbf{j} \mathbf{l k})$. This is shown in Fig. 2(a). Recursing to the next level, there are no primitive graphs which do not contain $\mathbf{i}, \mathbf{j}$, and $\mathbf{k}$, so the work on the $(\mathbf{i j})$ graph is complete.

Returning to the pivot $\mathbf{i}$, we now consider the (ijk) graph. At $\mathbf{j}$, we follow the same procedure as above. At $\mathbf{k}$, having excluded all graphs containing $\mathbf{i}$, and $\mathbf{j}$, only $(\mathbf{k l})$ remains, which we attach to $\mathbf{k}$. At $\mathbf{l}$ there are no graphs remaining to attach (Fig. 2(b)). The four-vertex graphs work in an analogous way, and are shown in Fig. 2(c).

The reasoning behind this algorithm is that at every point we must attempt to attach all possible graphs which would not lead to a graph which is accounted for elsewhere. At the $\mathbf{j}$ of the $(\mathbf{i j})$ graph, if any graph containing $\mathbf{i}$ were attached, say $(\mathbf{j} \mathbf{k i})$, traversing from our global pivot $\mathbf{i}$ would lead to a path $\mathbf{i j k i}$... which has already been accounted for under the (ijk) graph pivoted at $\mathbf{i}$.

The result of this algorithm on the four-vertex graph yields the rather unweildy graph shown in Fig. 4 (which has been computer generated). This has the corresponding expression (also computer generated) associated with it: 


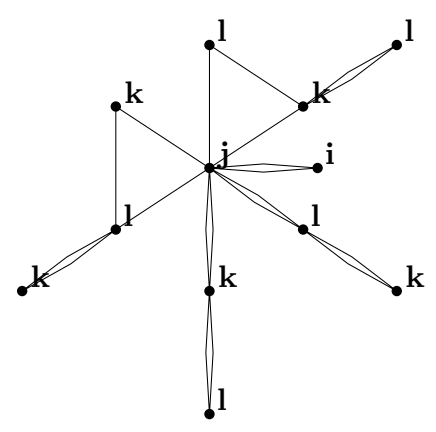

(a)

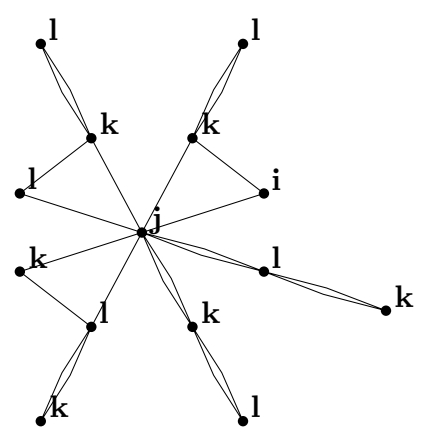

(b)

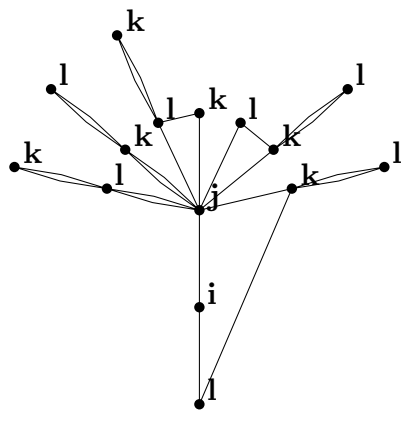

(c)

Fig. 2. The independent chains for a four-vertex graph beginning with (a) two-; (b) three- and (c) four-membered circuits

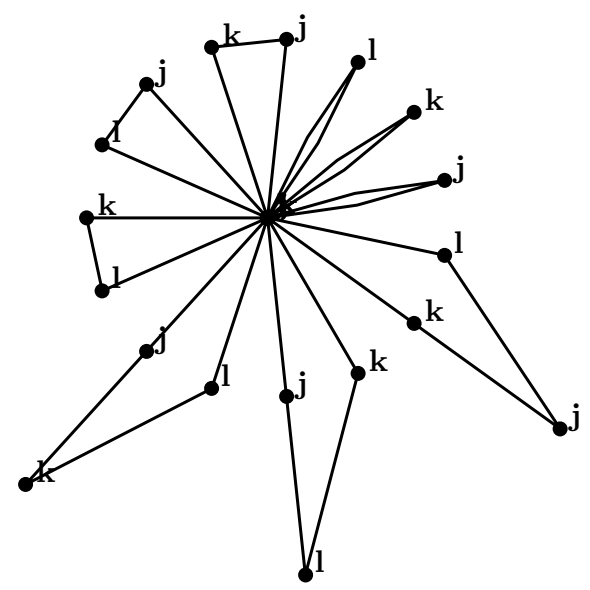

Fig. 3. All primitive circuits in the four-vertex graph which pivot at $\mathbf{i}$ joined in a star-graph 


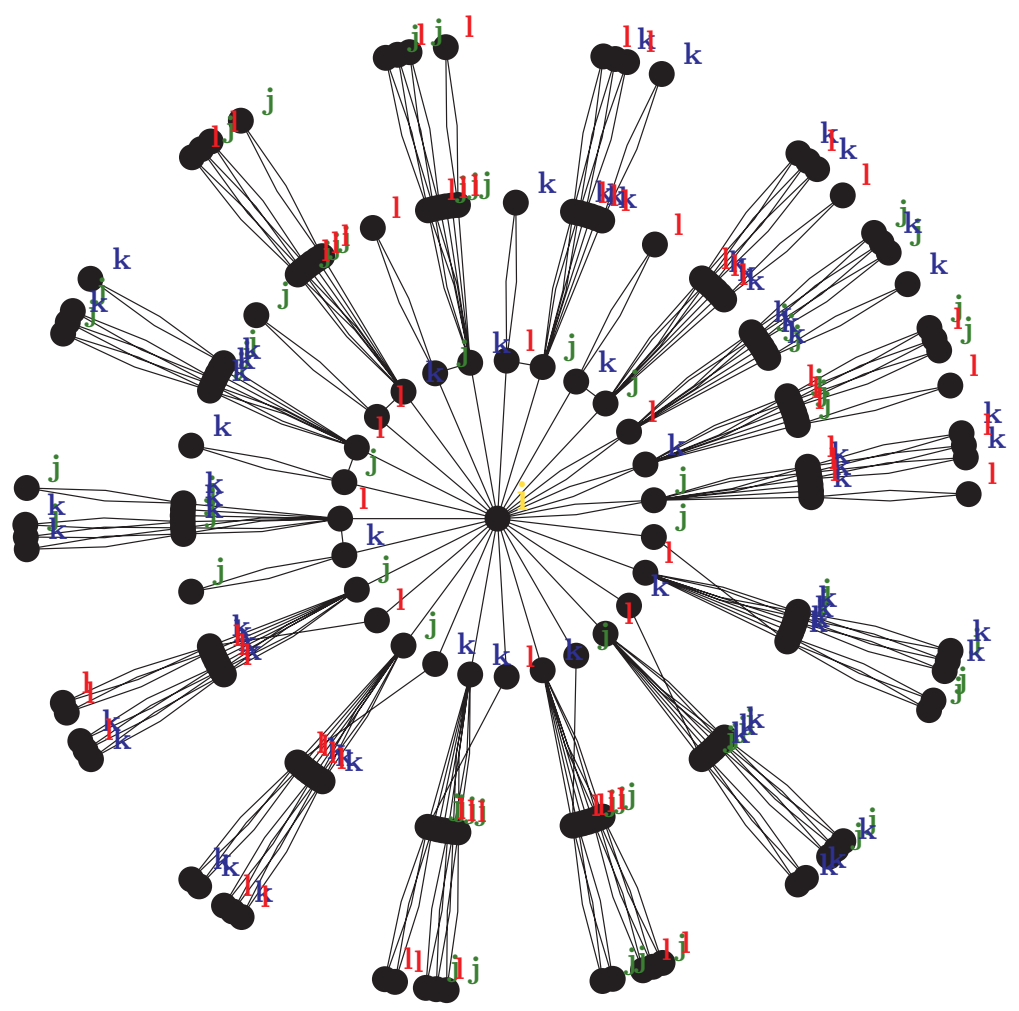

Fig. 4. The complete four-vertex graph of all independent circuits joined together and pivoted at $\mathbf{i}$

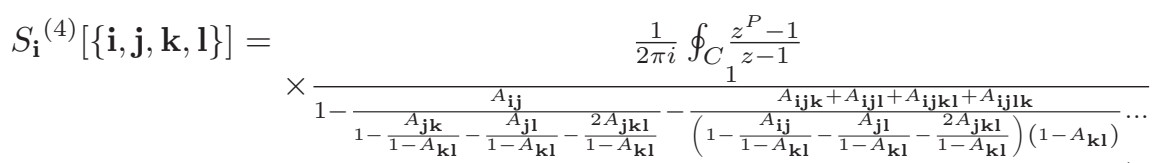

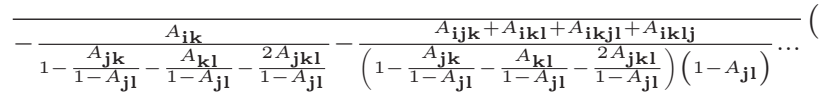

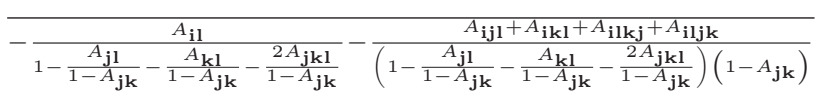

which simplifies to

$$
\begin{aligned}
& S_{\mathbf{i}}^{(4)}[\{\mathbf{i}, \mathbf{j}, \mathbf{k}, \mathbf{l}\}] \\
& =\frac{1}{2 \pi i} \oint_{C} \frac{z^{P}-1}{z-1} \frac{1-A_{\mathbf{j k}}-A_{\mathbf{j} \mathbf{}}-A_{\mathrm{kl}}-2 A_{\mathbf{j k} \mathbf{l}}}{1-A_{\mathrm{ij}}\left(1-A_{\mathrm{kl}}\right)-A_{\mathbf{i k}}\left(1-A_{\mathbf{j l}}\right)-A_{\mathbf{i l}}\left(1-A_{\mathbf{j k}}\right)-A_{\mathbf{j k}}-A_{\mathbf{j l}}-A_{\mathbf{k} \mathbf{l}}} \\
& -2 A_{\mathrm{ijk}}-2 A_{\mathrm{ijl}}-2 A_{\mathrm{ikl}}-2 A_{\mathrm{jkl}}-2 A_{\mathrm{ijkl}}-2 A_{\mathrm{ijlk}}-2 A_{\mathrm{ikjl}}
\end{aligned}
$$

The denominator reduces to a quartic polynomial in $z$, whose roots lead to four residues. 


\section{References}

1. A. J. W. Thom and A. Alavi (2005) A combinatorial approach to the electron correlation problem. J. Chem. Phys. 123, pp. 204106

2. R. P. Feynman (1948) Space-Time Approach to Non-Relativistic Quantum Mechanics. Rev. Mod. Phys. 20, pp. 367-387

3. R. P. Feynman, A. R. Hibbs (1965) Quantum Mechanics and Path Integrals, McGraw-Hill.

4. W. M. C. Foulkes, L. Mitas, R. J. Needs and G. Rajagopal (2001) Quantum Monte Carlo simulations of solids. Rev. Mod. Phys. 73, pp. 33-83

5. D. M. Ceperley (1992) Path-integral calculations of normal liquid-He-3. Phys. Rev. Lett. 69, pp. 331-334

6. For a comprehensive account of many quantum chemical methods see Modern Electronic Structure Theory by P. Jorgensen, J. Olsen and T. Helgaker (2000) Wiley, New York

7. J. B. Anderson (1975) Random-walk simulation of Schrodinger equation - H+3. J. Chem. Phys. 63, pp. 1499-1503; ibid. (1976) Quantum chemistry by randomwalk. 65, pp. $4121-4127$

8. A. Luchow and J. B. Anderson (1996) First-row hydrides: Dissociation and ground state energies using quantum Monte Carlo. J. Chem. Phys. 105, pp. $7573-7578$

9. M. E. Tuckerman and A. Hughes (1998) In "Classical and Quantum Dynamics in condensed systems", ed. B. J. Berne, G. Ciccotti, D. F. Coker, World Scientific

10. D. E. Knuth (1973) In "The Art of Computer Programming, Volume 1: Fundamental Algorithms", Addison Wesley

11. F. Becca, A. Parola, S. Sorella (2000) Ground-state properties of the Hubbard model by Lanczos diagonalizations. Phys. Rev. B 61, pp. R16287-R16290

12. R. W. Hall (2002) An adaptive, kink-based approach to path integral calculations. J. Chem. Phys. 116, pp. 1-7 